\title{
Ex vivo investigation of glycerol diffusion in skin tissue
}

\author{
Vadim D. Genin ${ }^{1 *}$, Daria K. Tuchina ${ }^{1}$, Ali Jaafar Sadeq ${ }^{1}$, Elina A. Genina ${ }^{1,2}$, Valery V. Tuchin ${ }^{1,2}$, \\ and Alexey N. Bashkatov ${ }^{1,2}$ \\ ${ }^{1}$ N.G. Chernyshevsky Saratov State University, 83 Astrakhanskaya Str., Saratov 410012, Russia \\ ${ }^{2}$ Tomsk State University, 36 Prosp. Lenina, Tomsk 634050, Russia \\ *e-mail: versetty2005@yandex.ru
}

\begin{abstract}
The change of optical parameters of rat skin ex vivo under the action of aqueous $30 \%, 50 \%, 60 \%, 70 \%, 85 \%$, and $100 \%$ glycerol solutions has been experimentally studied. The analysis of kinetics of the change of the studied parameters has allowed the estimate of the diffusion coefficient dependence upon the concentration of glycerol in the skin and the efficiency of optical clearing for each of the glycerol solutions. The results can find application in the development of new methods of noninvasive diagnostics and therapy of skin diseases. (C) 2016 Journal of Biomedical Photonics \& Engineering.
\end{abstract}

Keywords: glycerol, diffusion coefficient, skin, optical clearing.

Paper \#2933 received 2016.01.19 revised manuscript received 2016.03.10; accepted for publication 2016.03.10; published online 2016.03.29. doi: 10.18287/JBPE16.02.010303

\section{References}

1. V. V. Tuchin, Tissue optics: Light Scattering Methods and Instruments for Medical Diagnosis. Third edition, SPIE Tutorial Text in Optical Engineering, SPIE Press, Washington, Bellingham (2015). ISBN: 9781628415162

2. E. A. Genina, A. N. Bashkatov, and V. V. Tuchin, "Tissue optical immersion clearing," Expert Review of Medical Devices 7(6), 825-842 (2010).

3. D. Zhu, K. Larin, Q. Luo, and V. V. Tuchin, “Recent progress in tissue optical clearing," Laser \& Photonics Reviews 7(5), 732-757 (2013).

4. E. A. Genina, A. N. Bashkatov, Y. P. Sinichkin, I. Y. Yanina, and V. V. Tuchin, "Optical clearing of biological tissues: prospects of application in medical diagnostics and phototherapy," Journal of Biomedical Photonics \& Engineering 1(1), 22-58 (2015).

5. C. G. Rylander, O. F. Stumpp, T. E. Milner, N. J. Kemp, J. M. Mendenhall, K. R. Diller, and A. J. Welch, "Dehydration mechanism of optical clearing in tissue," J. Biomed. Opt. 11(4), 041117 (2006).

6. A. T. Yeh, and J. Hirshburg, "Molecular interactions of exogenous chemical agents with collagen-implications for tissue optical clearing," J. Biomed. Opt. 11(1), 014003 (2006).

7. E. A. Genina, A. N. Bashkatov, Y. P. Sinichkin, and V. V. Tuchin, "Optical clearing of skin under action of glycerol: ex vivo and in vivo investigations," Optics and Spectroscopy 109(2), 225-231 (2010).

8. M. A. Fox, D. G. Diven, K. Sra, A. Boretsky, T. Poonawalla, A. Readinger, M. Motamedi, and R. J. McNichols, "Dermal scatter reduction in human skin: a method using controlled application of glycerol," Lasers Surg. Med. 41, 251-255 (2009).

9. T. Son, and B. Jung, "Cross-evaluation of optimal glycerol concentration to enhance optical clearing efficacy," Skin Research and Technology 21(3), 327-332 (2015).

10. E. Youn, T. Son, H.-S. Kim, and B. Jung, "Determination of optimal glycerol concentration for optical tissue clearing," Proc. SPIE 8207, 82070J (2012).

11. J. B. Segur, and H. E. Oberstar, "Viscosity of glycerol and its aqueous solutions," Ind. and Eng. Chem. 43(9), 2117-2120 (1951).

12. A. N. Bashkatov, E. A. Genina, and V. V. Tuchin, "Measurement of glucose diffusion coefficients in human tissues," Chapter 19 in Handbook of Optical Sensing of Glucose in Biological Fluids and Tissues, V. V. Tuchin (Ed.), Taylor \& Francis Group LLC, CRC Press, 587-621 (2009). 
13. D. K. Tuchina, R. Shi, A. N. Bashkatov, E. A. Genina, D. Zhu, Q. Luo, and V. V. Tuchin, "Ex vivo optical measurements of glucose diffusion kinetics in native and diabetic mouse skin," J. Biophotonics 8(4), $332-346$ (2015).

14. Y. Nishijima, and G. Oster, "Diffusion of glycerol-water mixture," Bulletin of the Chemical Society of Japan 33(12), 1649-1651 (1960).

15. B. Chen, E. E. Sigmund, and W. P. Halperin, "Stokes-Einstein relation in supercooled aqueous solutions of glycerol," Phys. Rev. Lett. 96(14), 145502 (2006).

16. W. Chen, J. Jiang, W. Zhang, R. K. Wang, and K. Xu, "Typical application of skin diffusion optical model to quantitative description of tissue optical properties," Proc. SPIE 7176, 71760L (2009).

17. D. Huang, W. Zhang, H. Zhong, H. Xiong, X. Guo, and Z. Guo, “Optical clearing of porcine skin tissue in vitro studied by Raman microspectroscopy,” J. Biomed. Opt. 17(1), 015004 (2012).

18. J. Jiang, L. Zhang, and R. K. Wang, "Synergistic effect of hyperosmotic agents under topical application on optical clearing of skin tissue in vitro," Proc. SPIE 5696, 80-90 (2005).

19. Z. Mao, Z. Han, X. Wen, Q. Luo, and D. Zhu, "Influence of glycerol with different concentration on skin optical clearing and morphological changes in vivo," Proc. SPIE 7278, 72781T (2009).

20. X. Xu, and Q. Zhu, "Feasibility of sonophoretic delivery for effective skin optical clearing," IEEE Transactions on Biomedical Engineering 55(4), 1432-1437 (2008).

\section{Introduction}

Optical methods of diagnostics and treatment of different diseases are now widely used in medicine due to their relatively low cost and harmlessness for the patient's health. One of the important problems faced by the modern laser medicine is the transport of radiation to deeper tissue layers. Significant light scattering in tissues, particularly in skin, in the visible and nearinfrared spectral ranges seriously restricts the spatial resolution and probing depth of the used methods [1]. One of the possible ways to solve this problem is to reduce the light scattering by partial replacement of the interstitial fluid with various immersion agents, i.e., to make use of the so-called optical clearing technique [14].

The method of tissue optical clearing consists in matching the refractive indices of its components by partial replacement of the interstitial fluid with a biocompatible optical clearing agent (OCA) [1-4]. At present, three mechanisms are proposed to explain the reduction of light scattering under the effect of OCA, namely, the osmotic dehydration of the tissue, the replacement of interstitial fluid with the agent matching the refractive indices of the tissue components, and the structure modification or dissociation of the tissue collagen [1-4]. In real situations the first two mechanisms usually manifest themselves together. The difference, caused by the type of OCA and the tissue, will consist only in the relative contribution of each mechanism into the clearing effect. The influence of the third mechanism on the degree of clearing becomes noticeable only in the case of long-term action of hyperosmotic immersion liquids on the tissue [4].

The aqueous solutions of glycerol are immersion liquids widely used as OCAs due to their efficiency, availability, and biocompatibility [1-8]. Unfortunately, in spite of the wide use of glycerol for controlling the tissue optical parameters, there is no unambiguous answer to the question about the optimal concentration of the glycerol solution. Although the literature data [9,
10] allow the conclusion on the most efficient concentration of the glycerol solution from the point of view of the optical clearing degree, the concentration dependence of the glycerol diffusion coefficient in tissues remains to be an open issue.

The knowledge of the diffusion coefficients and the mechanisms of interaction of the studied OCAs with tissues are necessary for the development and optimization of the tissue optical clearing methods. Therefore, the aim of the present paper is to study the concentration dependence of the diffusion coefficient of glycerol in skin and the dependence of the skin optical clearing efficiency upon the glycerol concentration in the immersion clearing solution.

\section{Materials and methods}

As an object of study we used 60 samples of skin of white laboratory rats ex vivo (10 samples for each of the six clearing solutions). The hair was removed using the depilatory cream «Veet» (Reckitt Benckiser, France). The size of the samples was nearly $10 \times 15 \mathrm{~mm}^{2}$. The thickness of the samples was measured before the beginning of the experiment at five points by means of a micrometer with the accuracy $\pm 5 \mu \mathrm{m}$, the results were averaged.

As OCAs we used the aqueous 30\%, 50\%, 60\%, $70 \%, 85 \%$, and $100 \%$ solutions of glycerol, prepared by dissolving (in volume fractions) the dehydrated glycerol (REACHEM, Russia) with distilled water. The refractive indices of the agents, measured by means of the Abbe refractometer IRF-454-B2M (LOMO, Russia) at the wavelength $589 \mathrm{~nm}$, are presented in Table 1 . The measurement error is \pm 0.0001 .

The method of assessing the diffusion coefficients of immersion liquids in tissues, based on measuring the time dependence of collimated transmission in tissue samples, merged in the solution of clearing liquid, is thoroughly described in Refs. [12, 13]. 
Table 1 Refractive indices and viscosity of the glycerol solutions with different concentrations.

\begin{tabular}{ccc}
\hline $\begin{array}{c}\text { Concentration } \\
\text { of glycerol } \\
\text { solution }\end{array}$ & $\begin{array}{c}\text { Refractive } \\
\text { index of the } \\
\text { solution }\end{array}$ & $\begin{array}{c}\text { Viscosity of } \\
\text { aqueous solutions } \\
\text { of glycerol, } \\
\text { mPaxs [11] }\end{array}$ \\
\hline $30 \%$ & 1.378 & 2.5 \\
\hline $50 \%$ & 1.399 & 6 \\
\hline $60 \%$ & 1.415 & 10.8 \\
\hline $70 \%$ & 1.428 & 22.5 \\
\hline $85 \%$ & 1.452 & 109 \\
\hline $100 \%$ & 1.472 & 1410 \\
\hline
\end{tabular}

In the study of the interaction between the glycerol solutions and the skin samples it was assumed that only the refractive index of the interstitial fluid is changed due to the diffusion of the immersion liquid into the sample and the osmotic dehydration of the tissue. In the course of diffusion of the substance with the refractive index greater than that of the interstitial fluid into the tissue and the efflux of water from the tissue, the matching of the refractive indices of scatterers and interstitial fluid occurs, which leads to the reduction of the scattering coefficient of the tissue. Studying the kinetics of this process allows the estimation of the diffusion coefficient as a measure of the mean exchange flow rate of the hyperosmotic liquid into the tissue and of water from the tissue.

The maximal efficiency of the skin optical clearing (efficiency factor) was calculated using the formula

$$
O C_{e f f}=\frac{\mu_{t}(t=0)-\mu_{t}^{\min }}{\mu_{t}(t=0)},
$$

where $\mu_{t}$ is the attenuation coefficient calculated from the measured time dependence of the collimated transmission using the Bouguer-Lambert law; $\mu_{t}^{\min }$ is the minimal value of the attenuation coefficient.

The values of diffusion coefficient and the efficiency of optical clearing were calculated using the specially elaborated computer program for each wavelength and then averaged. The values of the diffusion coefficient were averaged over 10 samples for each of the studied concentrations of glycerol. The averaging of the optical clearing efficiency factor was also carried out over 10 samples for each of the studied concentrations of glycerol, but the values were grouped in three spectral regions, $500-600 \mathrm{~nm}, 600-700 \mathrm{~nm}$, and 700-900 $\mathrm{nm}$.

To measure the collimated transmission, we used the setup, consisting of the radiation source, namely, the halogen lamp HL-2000 (Ocean Optics, USA) (1), the optical fibres P400-1-VIS-NIR (Ocean Optics, USA), transporting the radiation to the tissue sample (2) and collecting the radiation passed through the sample (4), the cuvette with the sample (3), the spectrometer USB4000-Vis-NIR (Ocean Optics, USA) (5), and the computer (6) (Fig. 1).

For measuring the collimated transmission, the tissue samples were fixed in the plastic holder having the dimensions $38 \times 17 \mathrm{~mm}^{2}$ with the $8 \times 8 \mathrm{~mm}^{2}$ window and were put into the glass cuvette with $5 \mathrm{ml}$ of glycerol solution, placed between two optical fibres with the inner diameter $400 \mu \mathrm{m}$. To provide the beam collimation, the collimators 74-ACR (Ocean Optics, USA) were fixed at the faces of the fibres by means of the standard connectors SMA-905.

The kinetics of the collimated transmission variation was recorded by sequential recording of the transmission spectra in the range $500-900 \mathrm{~nm}$ every 3-5 minutes during 1-2 hours. All measurements were performed at room temperature $\left(\sim 20^{\circ} \mathrm{C}\right)$.

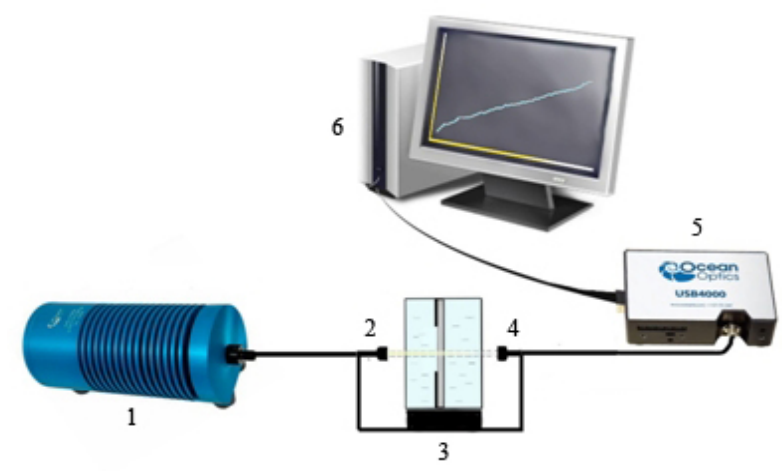

Fig. 1 Experimental setup: 1 - halogen lamp HL-2000; 2, 4 - optical fibres P400-1-VIS-NIR with collimators 74-ACR at the faces; 3 - cuvette with glycerol solution and skin sample, fixed in the frame; 5 - USB4000-VISNIR spectrometer; 6 - PC.

\section{Results and discussion}

Figure 2(a) presents a diagram illustrating the dependence of the diffusion coefficient of glycerol in skin upon the concentration of the glycerol solution. One can clearly see that the diffusion coefficient at first decreases till the concentration reaches $70 \%$ and then grows. This observation correlates well with the concentration dependence of the diffusion coefficient for glycerol in water [14] and is explained by both the increase of viscosity of the diffusing solution and the specific features of the formation of hydrodynamic radius of the diffusing molecules. As shown in Ref. [14], the hydrodynamic radius of glycerol molecules for the concentrations from 0 to $70 \%$ weakly depends on the glycerol concentration and decreases for higher concentrations, which is confirmed by the data of Ref. [15].

Thus, in the optical clearing of skin with the aqueous solutions of glycerol with the concentration $30-70 \%$ the reduction of the glycerol diffusion rate is due to the growth of the viscosity of the diffusing solution. For high-concentration glycerol solutions $(85-100 \%)$ there are two competing processes, the sharp increase of the diffusing solution viscosity and the decrease of the hydrodynamic radius of the glycerol molecules. As seen from Fig. 2(a), the contribution of the second process appears to be dominant, and we observe the growth of 


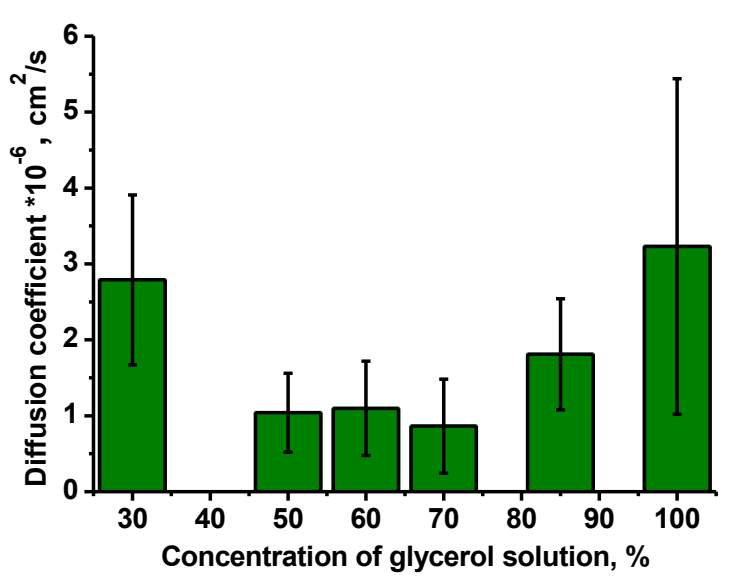

(a)

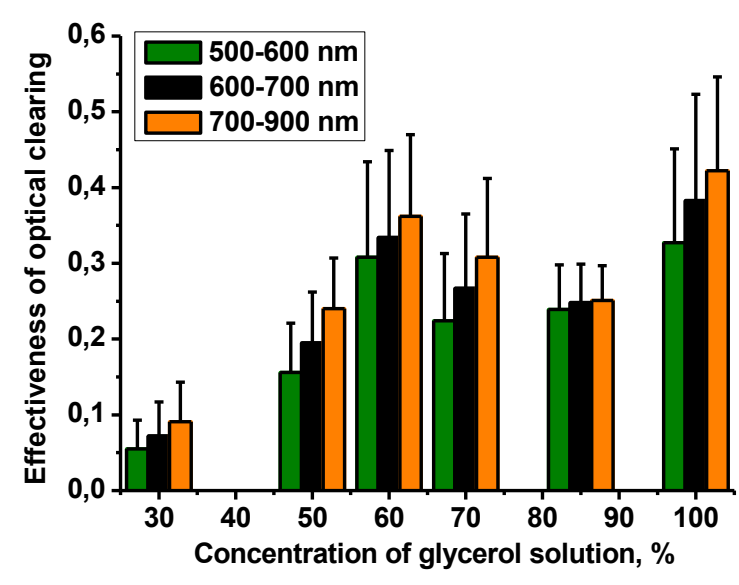

(b)

Fig. 2 Values of the diffusion coefficient (a) and optical clearing efficiency factor for the rat skin ex vivo in three different spectral ranges (b) depending on the concentration of solution. Bars correspond to the standard deviation.

Table 2 Values of the diffusion coefficient of glycerol in skin and the efficiency factor of the skin optical clearing.

\begin{tabular}{cccccc}
\hline $\begin{array}{c}\text { Glycerol solution } \\
\text { concentration }\end{array}$ & $\begin{array}{c}\text { Skin } \\
\text { samples } \\
\text { thickness, } \\
\mathbf{m m}\end{array}$ & $\begin{array}{c}\text { Efficiency of } \\
\text { optical clearing in } \\
\text { the region 500-600 } \\
\mathbf{n m}\end{array}$ & $\begin{array}{c}\text { Efficiency of } \\
\text { optical clearing in } \\
\text { the region 600-700 } \\
\mathbf{n m}\end{array}$ & $\begin{array}{c}\text { Efficiency of } \\
\text { optical clearing in } \\
\text { the region 700-900 } \\
\mathbf{n m}\end{array}$ & $\begin{array}{c}\text { Diffusion } \\
\text { coefficient, } \mathbf{c m}^{2} / \mathbf{s}\end{array}$ \\
\hline $30 \%$ & $0.60 \pm 0.08$ & $0.055 \pm 0.038$ & $0.072 \pm 0.045$ & $0.091 \pm 0.052$ & $(2.79 \pm 1.12) \times 10^{-6}$ \\
\hline $50 \%$ & $0.38 \pm 0.11$ & $0.156 \pm 0.065$ & $0.195 \pm 0.067$ & $0.240 \pm 0.067$ & $(1.04 \pm 0.52) \times 10^{-6}$ \\
\hline $60 \%$ & $0.32 \pm 0.10$ & $0.308 \pm 0.126$ & $0.334 \pm 0.115$ & $0.362 \pm 0.108$ & $(1.098 \pm 0.62) \times 10^{-6}$ \\
\hline $70 \%$ & $0.48 \pm 0.07$ & $0.224 \pm 0.089$ & $0.267 \pm 0.098$ & $0.308 \pm 0.104$ & $(0.86 \pm 0.73) \times 10^{-6}$ \\
\hline $85 \%$ & $0.57 \pm 0.16$ & $0.239 \pm 0.059$ & $0.248 \pm 0.051$ & $0.251 \pm 0.046$ & $(1.81 \pm 1.13) \times 10^{-6}$ \\
\hline $100 \%$ & $0.46 \pm 0.13$ & $0.327 \pm 0.124$ & $0.383 \pm 0.14$ & $0.422 \pm 0.124$ & $(3.23 \pm 2.21) \times 10^{-6}$ \\
\hline
\end{tabular}

the diffusion coefficient. Besides, as mentioned above, the coefficient of diffusion measured in the present work is a measure of the mean rate of the total exchange flow of the hyperosmotic liquid (glycerol solution) into the tissue and the water from the tissue. Correspondingly, for more correct determination of the diffusion coefficient of glycerol in the tissue one has to separate these processes from each other, which will be the aim of our further studies.

Table 2 presents the calculated diffusion coefficients for each of the glycerol solutions, as well as the efficiency coefficients of the skin optical clearing, grouped over three spectral ranges. Large values of the standard deviation for the determined values of the diffusion coefficients are due to the natural spread of the optical, structural, and morphological parameters of the skin samples.

Figure 2(b) shows the dependence of the skin optical clearing efficiency upon the concentration of glycerol solutions. From Fig. 2(b) it follows that the larger value of the OCA diffusion coefficient does not ensure the higher efficiency of the tissue optical clearing. Among the studied OCAs, in spite of the high rate of penetration into the tissue, the lowest efficiency of the optical clearing is observed in the $30 \%$ glycerol solution. This is because although the 30\% glycerol solution is a liquid with the minimal viscosity, it also possesses the minimal refractive index.

The growth of the efficiency coefficient of the skin optical clearing in the case of using the glycerol solutions with concentrations of $30-60 \%$ as OCAs is explained by the increased matching of the OCA refractive index $(1.378$ for $30 \%$ solution and 1.415 for $60 \%$ solution) to that of the collagen fibres (1.411-1.416 [1]), which are the main scatterers in the skin. Then the efficiency of optical clearing decreases because the refractive index of OCA becomes greater than that of the collagen fibres. These results correlate well with the data, presented in Refs. [9, 10, 16-20], where the optical transmission of skin increased with the growth of the concentration of glycerol solution in the ranges $30-80 \%$ [16], 40-80\% [17], 50-80\% [18], 20-55\% [19], 40-80\% [20].

Similar results are demonstrated also in Refs. [9, 10], where the glycerol solution concentrations from 50 to $90 \%$ were used, and it was shown that the maximal efficiency of clearing is observed for the $70 \%$ solution, while for smaller or greater concentrations the clearing efficiency significantly decreases.

For the concentrations of the glycerol solution from $85 \%$ to $100 \%$ the efficiency of optical clearing increases again. Probably, this fact is due to the dominant role of 
the dehydration mechanism of the optical clearing: the dehydration of the skin leads to the reduction of its thickness and, therefore increases the collimated transmission. Probably, the refractive index of collagen fibres is increased due to the destruction of their hydrate shells, leading to better matching of refractive indices of skin scatterers and their environment, the role of which in this case is played by the nigh-concentration glycerol solution. However, this hypothesis requires further studies.

\section{Conclusion}

The obtained results allow one to estimate the efficiency of using aqueous glycerol solutions with different concentrations as clearing agents controlling the scattering properties of skin. The most efficient optical clearing within the spectral range 500-900 nm was demonstrated with the $60 \%$ and $100 \%$ solutions of glycerol. Sufficiently high diffusion rate in combination with high efficiency of optical clearing was demonstrated by the $85 \%$ solution of glycerol.

\section{Acknowledgments}

The work was carried out under the financial support from the Russian Science Foundation, grant No. 14-1500186 . 